Research Paper

\title{
Risk factors for cervical intraepithelial neoplasia and cervical cancer in Chinese women: large study in Jiexiu, Shanxi Province, China
}

\author{
Zhilian Wang1*; Jintao Wang2*; Jinrong Fan ${ }^{3 *}$; Weihong Zhao1; Xin Yang1; Lingmei $\mathrm{Wu}^{3}$; Dongyan Li1; ling \\ Ding2; Wei Wang1; Junjie Xu1; Michelle Stram; Chengquan Zhao ${ }^{4}$; Min Hao ${ }^{1 凶}$ \\ 1. Department of Obstetrics and Gynecology, Second Hospital of Shanxi Medical University, Taiyuan, China. \\ 2. Department of Epidemiology, School of Public Health, Shanxi Medical University, Taiyuan, China. \\ 3. Jiexiu Family Planning/Women and Children Health Service Center, Jinzhong, China. \\ 4. Department of Pathology, Magee-Womens Hospital, University of Pittsburgh Medical Center. 300 Halket St, Pittsburgh, PA, 15213, USA. \\ * These authors contributed equally to this work. \\ $\square$ Corresponding author: Min Hao, MD. Department of Obstetrics and Gynecology, The Second Hospital of Shanxi Medical University, 382 Wuyi Rd, Taiyuan, \\ China. Email address: 2yuanhaomin@163.com; Fax: +86 3513362866
}

( ) Ivyspring International Publisher. This is an open access article distributed under the terms of the Creative Commons Attribution (CC BY-NC) license (https://creativecommons.org/licenses/by-nc/4.0/). See http://ivyspring.com/terms for full terms and conditions.

Received: 2016.08.31; Accepted: 2016.12.22; Published: 2017.03.12

\begin{abstract}
We aimed to investigate the risk factors for cervical intraepithelial neoplasia $(\mathrm{CIN})$ in Jiexiu, Shanxi Province, China. Twenty thousand eligible married women (age: 18-65 years) were administered with a questionnaire on potential risk factors for $\mathrm{CIN}$ and underwent liquid based Pap test. All women with abnormal cytological results underwent colposcopy with biopsy. Based on the biopsy pathology results, women were then assigned to either study group (with CIN) or control group (negative for histological results and volunteered to participate in the follow up study). The women in both study group and control group underwent vaginal microflora detection and dietary survey. The potential risk factors were analyzed by using ordinal logistic regression. Among the 20,000 women ne 1,438 women (7.19\%) had cytologic abnormalities and $410(2.05 \%)$ women were diagnosed histologically with CIN lesions, including 317 (1.58\%) with CINI, 93 (0.50\%) with CIN2/3and $11(55 / 100,000)$ with squamous cell carcinoma (SCC). The average daily dietary folate intake was significantly lower in the study group $(344.61 \pm 153.07 \mu \mathrm{g})$ than in the control group $(371.50 \pm 166.58 \mu \mathrm{g} ; \mathrm{P}<0.001)$. Multivariate analysis demonstrated that age of $56-65$ years, farming as the husband's occupation, unwashing the vulva after sexual intercourse, and low self-reported folate intake were positively associated with $\mathrm{CIN}$ development and might have contribution to the increased CIN incidence in this population. These findings may provide help to develop the strategies to reduce the risk of cervical cancer in China.
\end{abstract}

Key words: CIN, cervical cancer, risk factors, folate, China

\section{Introduction}

Due to the development and widespread use of cervical cancer screening tools, the incidence of cervical cancer has declined worldwide. ${ }^{1}$ Moreover, the detection rate of cervical intraepithelial neoplasia (CIN) has increased.2-4 Cervical cancer remains an important global public health problem. More than $85 \%$ of cases of cervical cancer occur among women in the less-developed countries. China with the world's largest population accounts for $14 \%$ of the global burden of new cervical cancer cases annually. 5 Furthermore, the reported incidence of cervical cancer in China (9.6 per 100,000 women) may be underestimated. ${ }^{5}$ Chinese data from 2003 to 2010 indicates that cervical cancer incidence and mortality have increased in the 21st century. ${ }^{6}$ Among all the provinces in China, the morbidity and mortality rates 
of cervical cancer were higher in Shanxi Province. In particular, the mortality rate of cervical cancer is 23.04 per 100,000 women in Yangcheng County, Shanxi Province, ${ }^{7} 10$-fold higher than that in other areas of China. ${ }^{8,9} \mathrm{~A}$ strategy to reduce the incidence of cervical cancer is certainly necessary. Before the strategy is developed, the risk factors and other relevant dynamics that are potentially associated with CIN lesions need to be determined. The recognition of these contributory factors will provide an opportunity to develop and implement interventions tailored to address the occurrence and progression of CIN.

Previous studies have attempted to determine the incidence of CIN in China. The incidence of CIN was reportedly $0.46 \%$ in Jingan County, Jiangxi Province, in $2010^{8}$ and $0.12 \%$ in Beijing in 2014. ${ }^{10}$ However, the variation in the incidence of CIN in different regions of China has not been fully characterized. Moreover, the influence of the geographical features on the incidence of CIN and cervical cancer has not been determined in Jiexiu, Shanxi Province, China.

The population in the Taihang Mountain area in Shanxi Province reportedly has a high incidence of cervical cancer. A previous study suggested that folate deficiency might be associated with an increased risk of cervical cancer through a synergistic action with the human papillomavirus (HPV) infection. ${ }^{11}$ However, no large study has been reported. We aimed to assess the relevant epidemiological and cervical cancer screening data, including lifestyle, dietary status, sexual habits, and reproductive conditions of married women residing in the Jiexiu area of Shanxi Province. We hope to determine the risk factors and local geographical features associated with $\mathrm{CIN}$, and elucidate fundamental reference data for subsequent studies on the relationship of folate intake with CIN and cervical cancer.

\section{Materials and Methods}

\section{Objectives}

Twenty thousand women were selected from 82 villages, towns, and mining regions in the Jiexiu area, Shanxi Province, China, between June 2014 and November 2014. All the women volunteered to participate in the study. The selection criteria were as follows: married women aged 18-65 years, women of the Han ethnicity, and women who had resided in Jiexiu for at least 1 year. The exclusion criteria were as follows: pregnancy, history of hysterectomy or cervical conization, current or prior malignancy, hematologic or gastrointestinal disease, and the use of $B$ vitamin supplementation within the past 3 months.

\section{Study design}

The study was conducted in 2 phases: a baseline (cross-sectional) phase and a two-year follow-up (longitudinal) phase. The data reported in this article included the results from only the baseline phase because the follow-up study in phase 2 is ongoing. All the subjects had volunteered to participate in the study and signed informed consent. The study protocol and informed consent form were reviewed and approved by the Ethics Committee of Second Hospital of Shanxi Medical University, and was registered in the Chinese Clinical Trial Register (registration number: ChiCTR-ROC-15006479). Participating women underwent one or two study visits at baseline, as follows.

Study visit 1: Enrollment visit for all participants. The 20,000 eligible women were administered a questionnaire on the potential risk factors for CIN and underwent the Pap test using liquid based cytology analysis.

\section{$\mathrm{CIN}$ questionnaire}

A close-ended questionnaire was created by the members of our research group (an epidemiologist and a gynecological oncologist) based on literature reviews, expert evaluations, and a small-scale pre-survey, to evaluate the potential factors related to the development of CIN. Each participant completed the questionnaire at the time of enrollment. The participants were asked for the questions in a face-to-face interview by trained and qualified investigators. The questionnaire included five primary domains: a) participant characteristics including age, education, employment, spouse employment, marital status, income, and smoking habits of the participants and the spouse when applicable; b) vaginal hygiene including frequency of vulva cleaning, frequency of washing the vulva after sexual intercourse, frequency of washing underwear, bathing facilities, and method of bathing; c) gynecologic history including menstrual and obstetrical history, sexual history, and contraceptive use; d) medical history; and e) family history of cancer.

\section{Pap test}

All Pap tests were prepared by using liquid based cytology (LBC) method. The cytological evaluation was performed by two cytopathologists at the Second Hospital of Shanxi Medical University. Pap tests were reported by using the Bethesda System (TBS) 2001 terminology. ${ }^{12}$ The cases with an interpretation of atypical squamous cells of undetermined significance (ASC-US) or worse were further reviewed by a senior cytopathologist blindly. 
$10 \%$ cases with the negative test were reviewed by another pathologist for quality control.

Study visit 2: Colposcopy visit was performed for the selected participants. Vaginal microflora detection and dietary survey were done also.

All women with abnormal Pap test results (ASC-US or worse) were referred for colposcopy with biopsy and/or endocervical curettage (ECC) according to the same protocol. The women with negative or inadequate colposcopic findings also underwent ECC. The women were divided into the study group and the control group based on the histological findings. The study group consisted of the women with histological CIN1, CIN2/3, whereas the control group consisted of the women with negative histological results who volunteered to participate in the follow-up study. Vaginal microflora detection and dietary survey were conducted for these women.

\section{Laboratory testing methods}

\section{Cervical Pap cytology Specimen Collection}

All participants were asked to abstain from sexual intercourse and not to perform vaginal lavage or take medications for 48 hours prior to the sampling. At the visit1, all participants underwent a routine pelvic examination by gynecologists and the cervical LBC specimen was obtained (Lituo Biotechnology Co.Ltd., Hunan, China). If the collected cytological samples were inadequate for Pap test, re-sampling was done until satisfactory specimens were obtained. At the visit 2, the vaginal fluid specimen was obtained for microflora detection before colposcopy examination.

\section{Cytology testing}

The LBC slide was prepared according to the manufactures' protocol (Lituo Biotechnology Co, Hunan, China; http://www.lituo.com.cn). in the cytology laboratory at Jiexiu Family Planning/Women and Children Health Service Center. The samples were fixed within an ethanol-based preservative solution, and the filter method was used for preparation of the slides. An LTSYJ 2000 automated slide Preparation Unit (Lituo Biotechnology) was used in this cytology laboratory.

\section{Vaginal Microflora Detection}

The vaginal fluid samples were collected by using culture swab. Two vaginal smear slides were made for the detection of vaginal microflora. One saline wet slide was viewed directly under an optical microscope to assess the cleanliness, the presence of trichomoniasis and fungal hyphae. The other slide with Gram-stain was observed under a microscope for determining bacterial density, bacterial diversity, and predominant bacterial flora. The diagnosis of bacterial vaginosis (BV) was based on the criteria described by Nugent et al. ${ }^{13}$ Vaginal microbial function was examined by using the vaginitis automatic detection workstation and the vaginitis multi test kit by dry chemical enzymatic detection (model: bPR-2011A, Jiangsu Bioperfectus Technologies Co. Ltd., Taizhou, Jiangsu, China) The detection items included $\mathrm{H}_{2} \mathrm{O}_{2}$, neuraminidase, leukocyte esterase, $\beta$-glucuronidase, acetylglucosaminidase, and $\mathrm{pH}$ value. Finally, we referred to Dr. Fu's standard for the diagnosis of vaginitis. ${ }^{14}$

\section{Colposcopy and cervical histological examination}

Colposcopy was performed by gynecological specialists at the Second Hospital of Shanxi Medical University according to a standard protocol, within 12 weeks from the Pap test. Video colposcopy was used the SLC-2000 device (Shenzhen Goldway Company, Shenzhen, Guangdong, China). During the colposcopy, the cervix was divided into quadrants, and each quadrant was examined. All visually abnormal areas were biopsied, and the quadrants without a visible lesion were biopsied at the squamo-columnar junction ("random biopsy"). Women with abnormal cytology results and negative or inadequate colposcopic findings also underwent endocervical curettage (ECC). The cervical biopsy and ECC were evaluated by two gynecological pathologists at the Second Hospital of Shanxi Medical University. The cases were classified as negative, CIN1, CIN2, CIN3, adenocarcinoma in situ or carcinoma. ${ }^{15}$ The pathologists would not know the Pap test results while they read the cervical biopsy or ECC tissue specimens. If the diagnoses were discordant between the two pathologists, the cases would be reviewed by the third senior pathologist. The three pathologists would review the cases together to arrive a consensus diagnosis for some difficult or equivocal cases.

\section{Dietary folate intake questionnaire}

Our research group, including an epidemiologist and nutrition experts, designed and administered a food frequency questionnaire (FFQ) for this investigation, based on the FFQ designed by Wang ${ }^{16}$ and while considering the eating habits of the local people through literature reviews, expert evaluations, and a small-scale pre-investigation survey. This questionnaire had been applied in a previous study, ${ }^{11}$ which demonstrated that data on dietary habits could be successfully collected with this tool. Twenty-four types of food were analyzed, including common local cereals, pulses, fresh vegetables, fresh fruits, meat, 
protein, and dairy products, by taking into account the seasonal variability. We estimated the dietary folate intake averaged daily by using data on the self-reported intake of the 24 types of foods in the questionnaire based on the China Food Composition Table 2002. ${ }^{17}$

\section{Statistical Analysis}

All statistical analyses were conducted using the Statistical Package for the Social Sciences (SPSS) version 13.0 software (SPSS Inc, Chicago, IL, USA). The quantitative variables (normally distributed) were described using mean and standard deviations $(\mathrm{M} \pm \mathrm{SD})$, and were compared between groups by using analysis of variance. The quantitative variables with a skewed distribution data (daily dietary folate intake levels) were expressed as median and interquartile range $(\mathrm{M} \pm \mathrm{QR})$, and comparisons between the groups was performed using the Wilcoxon test. Qualitative variables were expressed as percentages, and differences between the groups were statistically analyzed using the chi-square test, as well as the chi-square test for trends. Statistical tests were two-sided, and a $\mathrm{P}$ value of $<0.05$ was considered statistically significant. Prevalence estimates of cytological results were calculated based on all eligible women with valid Cytology test results. Crude prevalence estimates of cervical disease were calculated based on women who underwent colposcopy / biopsy. The 95\% confidence intervals (CIs) were computed for CIN, whereas the $95 \%$ CI for the Poisson processes were also calculated based on the results of CIN diagnosed by histological result. As $<20$ cases of SCC were diagnosed by histological result, the $95 \%$ CI for SCC was determined through the look-up table method. The potential risk factors for CIN were analyzed using ordinal logistic regression. Variables with a $\mathrm{P}$ value of $<0.05$ in the univariate analysis were included in the multivariate model.

\section{Results}

\section{CIN Questionnaire}

\section{Basic information}

Twenty thousand women aged $44.09 \pm 9.473$ years were included in this study. Of these women, 12,110 $(60.55 \%)$ had received education in junior middle school or below, whereas 7890 (39.45\%) had completed senior middle school or higher education. Most women were either housewives $(6648,32.24 \%$ ) or farmers $(6583,33.92 \%)$. All 20,000 women were married and 962 women $(4.81 \%)$ were divorced, widowed, or separated at the time of study. The majority of married women had a husband whose occupation was either farming $(6871,34.36 \%)$ or factory work $(4878,24.39 \%)$. The average annual family income was $31,658.33 \pm 23,166.81$ Chinese Yuan Renminbi (RMB) (range, 0-600,000). Three hundred forty seven women $(1.71 \%)$ did not have any family income, and 568 women (2.84\%) had an annual family income of less than $5000 \mathrm{RMB}$. Two hundred fifty nine women $(1.29 \%)$ were smokers and $12,045(60.23 \%)$ women had husbands who were smokers.

\section{Vaginal hygiene and reproductive factors}

The frequency of vulva washing was reported as less than once a week by 1,472 women $(7.36 \%), 2-3$ times per week by 18,521 women $(92.60 \%)$, and $>3$ times a week by 7 women $(0.04 \%)$. Fifteen thousand seven hundred ninety eight women (78.99\%) washed their vulva after sexual intercourse. The average age at marriage was $23.33 \pm 2.371$ years (range, $17-42$ ). The average age at first sexual intercourse was $23.31 \pm 2.585$ years (range, 11-42). The average primiparous age was 24.62 2.609 years (range, 17-54). Contraceptive measures were adopted by 18,911 (93.48\%) women, including 8133 women $(40.7 \%)$ with intrauterine devices and 7726 women (38.19\%) with tubal ligation. Four thousand nine hundred forty women $(24.42 \%)$ had reached menopause.

\section{Family history of cancer}

A family history of cancer, defined as reported cancer in the immediate family over the previous three generations, was observed in 2,228 women $(11.14 \%)$, including 1,188 women with a paternal history of cancer and 1,040 with a maternal history of cancer. Two hundred and three women $(1.02 \%)$ had a family history of cervical cancer.

\section{Cervical Pap cytology results}

Among 20,000 women, 125 women (0.63\%) had an unsatisfactory Pap test initially. These patients underwent repeat sampling to obtain the satisfactory results. Overall, 1,438 (7.19\%) women showed abnormal Pap cytology results, including 1,270 women $(6.35 \%)$ with ASC-US, 7 women $(0.04 \%)$ with atypical squamous cells, cannot exclude high-grade squamous intraepithelial lesion (ASC-H), 125 women $(0.63 \%)$ with low-grade squamous intraepithelial lesion (LSIL), 32 women $(0.16 \%)$ with high-grade squamous intraepithelial lesion (HSIL), 2 women $(0.01 \%)$ with squamous cervical carcinoma (SCC), and 2 women $(0.01 \%)$ with atypical glandular cells (AGC). A total of 18,562 women $(92.81 \%)$ women tested negative for intraepithelial lesion or malignancy (NILM) (Table 1). 
Table 1. Cervical cytological results

\begin{tabular}{ll}
\hline TBS Category & No $(\%)$ \\
\hline ASC-US & $1270(6.35)$ \\
ASC-H & $7(0.04)$ \\
LSIL & $125(0.63)$ \\
HSIL & $32(0.16)$ \\
AGC & $2(0.01)$ \\
SCC & $2(0.01)$ \\
NILM & $18,562(92.81)$ \\
Total & $20,000(100.00)$ \\
\hline
\end{tabular}

TBS, the Bethesda system; ASC-US, atypical squamous cells of undetermined significance; ASC- $\mathrm{H}$, atypical squamous cells, cannot exclude high-grade squamous intraepithelial lesion; LSIL, low-grade squamous intraepithelial lesion; HSIL, high-grade squamous intraepithelial lesion; AGC, atypical glandular cells; SCC, squamous cervical carcinoma; NILM, negative for intraepithelial lesion or malignancy.

\section{Colposcopy and cervical histological examination results}

Colposcopy with biopsy was performed in all 1438 women with abnormal Pap cytology results. Adequate colposcopy examination was performed in 889 women, whereas 549 women had an inadequate colposcopy examination and subsequently underwent ECC. The biopsy was taken at the suspicious areas, and random biopsy was taken at the squamo-columnar junction in the cases where no visible lesions were noted in any quadrant.

Overall, 421 (2.05\%; 95\%CI, 1.85-2.25) women were diagnosed with CIN1 and above lesions, including 317 (1.58\%; 95\% CI, 1.41-1.76) women with CIN1, 93 (0.50\%; 95\% CI, 0.37-0.56) with CIN2/3, and 11 (95\% CI, 27-99) with SCC. One hundred and four women $(0.52 \%$; 95\% CI, 0.42-0.62) were diagnosed with CIN2 and above lesions (CIN2+). Of the 1270 women with ASC-US Pap test, 273 (21.49\%) were diagnosed with CIN1 and 90 (7.05\%) were diagnosed with CIN2+, including 81 with CIN2/3 and 9 with SCC.

\section{Vaginal microflora detection results}

There were no significant differences between the study groups (CIN1 and CIN2/3) and the control group in terms of the detection rates of trichomonas vaginitis, vulvovaginal candidiasis, bacterial vaginosis, and other types of vaginitis $(\mathrm{P}<0.05)$ (Table 2).

\section{Dietary folate intake}

The average amount of daily dietary folate intake was $344.61 \pm 153.07 \mu \mathrm{g}$ for the 410 women with CIN lesions in study group, $371.50 \pm 166.58 \mu \mathrm{g}$ for the 626 women in the control group. The difference of the amount of daily folate intake was statistically significant $(\mathrm{P}<0.001)$ (Table 3$)$.
Table 2. Detection results of vaginitis in the study and control groups $(n=1036)$

\begin{tabular}{|c|c|c|c|c|c|c|}
\hline Group & $\mathrm{N}$ & $\begin{array}{l}\text { Trichomonas } \\
\text { vaginitis (n, } \\
\%)\end{array}$ & $\begin{array}{l}\text { Bacterial } \\
\text { vaginosis } \\
(\mathrm{n}, \%)\end{array}$ & $\begin{array}{l}\text { Vulvovaginal } \\
\text { candidiasis } \\
(\mathrm{n}, \%)\end{array}$ & $\begin{array}{l}\text { Other } \\
\text { types of } \\
\text { vaginitis } \\
(n, \%)\end{array}$ & $\begin{array}{l}\text { Total } \\
(\mathrm{n}, \%)\end{array}$ \\
\hline $\begin{array}{l}\text { Control } \\
\text { group }\end{array}$ & 626 & $186(29.71)$ & $15(2.40)$ & $13(2.08)$ & $54(8.63)$ & $268(42.81)$ \\
\hline $\begin{array}{l}\text { CIN1 } \\
\text { group }\end{array}$ & 317 & 108 (34.07) & $5(1.58)$ & $8(2.52)$ & $16(5.05)$ & $137(43.22)$ \\
\hline $\begin{array}{l}\text { CIN2/3 } \\
\text { group }\end{array}$ & 93 & $38(40.86)$ & $3(3.23)$ & $1(1.08)$ & $12(12.90)$ & $54(58.06)$ \\
\hline $\begin{array}{l}\text { Total } \\
\chi^{2}=7.853, \\
P=0.020\end{array}$ & 1036 & $332(32.05)$ & $23(22.20)$ & $22(2.21)$ & $82(7.92)$ & $459(44.31)$ \\
\hline
\end{tabular}

Table 3. Daily dietary folate intake level in the study and control groups $(n=1036)$

\begin{tabular}{lllll}
\hline Groups & No & Dietary folate intake $(\mu \mathrm{g})^{*}$ & $\mathrm{Z}$ & P value \\
\hline Control & 626 & $371.50 \pm 166.58$ & -3.989 & $<0.001$ \\
Study & 410 & $344.61 \pm 153.07$ & & \\
\hline * Values are presented as median \pm quartile range & &
\end{tabular}

\section{Analysis of the Potential Risk Factors for CIN}

\section{Univariate analysis}

The following variables differed significantly between the study group (CIN group) and the control group in the univariate analysis: age, education level, occupation, husband's occupation, washing the vulva after sexual intercourse, use of tubal ligation for contraception, duration after tubal ligation, and self-reported average daily dietary folate intake $(\mathrm{P}<0.05 ;$ Table 4). The factors that did not differ significantly between the control and CIN groups, included: the marital status, annual income, smoking status of the participant and spouse, bathing facilities, bathing frequency, method of bathing, frequency of genital washing, frequency of washing underwear, sexual intercourse during menstruation, the type of menstrual sanitary products used, reproductive factors including the age at menarche, menstrual cycle, age at first intercourse, primiparous age, age at first birth, parity, sexually transmitted diseases in the participant and spouse, presence of vaginitis, use of contraceptive pills or an intrauterine contraceptive device, medical history (such as whether prior cervical cancer screening had been performed), and family history of cancer.

\section{Multivariate analysis}

The significant variables in the univariate analysis were examined in the multivariate analysis, including age, husband's occupation, washing the vulva after sexual intercourse, and average daily dietary folate intake. The 56-65-year-old group had the highest risk of CIN, whereas the CIN risk in the 
$36-45$-year-old group was only $61.4 \%$ of that in the 56-65-year-old group $(\mathrm{P}=0.006)$. Women whose husband's occupation was farming had the highest risk of CIN. The CIN risk in women with husbands who worked as medical, scientific, or educational staff was $62.19 \%$ of that in women with husbands who were farmers $(\mathrm{P}=0.025)$. The $\mathrm{CIN}$ risk in women who did not wash their vulva after sexual intercourse was 1.831 times greater than that of women who did
$(\mathrm{P}<0.001)$. For each $1-\mu \mathrm{g}$ increase in the average daily dietary folate intake, the risk of CIN was reduced by $0.001 \%(\mathrm{P}<0.001)$. Accordingly, multivariate analysis showed that age between 56 and 65 years, farming as the husband's occupation, not cleaning the vulva after sexual intercourse and low level of dietary folate intake were potential risk factors for the development of CIN (Table 5).

Table 4. Univariate analysis of the potential risk factors for $\mathrm{CIN}$. ( $\mathrm{n}=1036,410$ women with $\mathrm{CIN}$ lesions and 626 women without $\mathrm{CIN}$ lesion)

\begin{tabular}{|c|c|c|c|c|c|}
\hline Variable & B & S.E. & Wald & OR $(95 \%$ CI) & $\mathrm{P}$ \\
\hline \multicolumn{6}{|l|}{ Age(years) } \\
\hline $56-65$ & & & & 1.000 & \\
\hline $46-55$ & -0.405 & 0.172 & 6.724 & $0.667(0.491-0.909)$ & 0.010 \\
\hline $36-45$ & -0.434 & 0.156 & 6.408 & $0.648(0.463-0.907)$ & 0.011 \\
\hline$\leq 35$ & -0.262 & 0.225 & 1.360 & $0.769(0.496-1.195)$ & 0.24 \\
\hline \multicolumn{6}{|l|}{ Education level } \\
\hline Senior middle school or above & & & & 1.000 & \\
\hline Junior middle school or below & 0.309 & 0.128 & 5.789 & $1.362(1.059-1.752)$ & 0.016 \\
\hline \multicolumn{6}{|l|}{ Occupation } \\
\hline Farmer & & & & 1.000 & \\
\hline Worker & -0.429 & 0.225 & 3.619 & $0.651(0.418-1.013)$ & 0.057 \\
\hline Medical, scientific, educational staff & -0.473 & 0.193 & 5.999 & $0.623(0.427-0.910)$ & 0.014 \\
\hline Other & -0.264 & 0.148 & 3.165 & $0.768(0.574-1.027)$ & 0.057 \\
\hline \multicolumn{6}{|l|}{ Husband's occupation } \\
\hline Farmer & & & & 1.000 & \\
\hline Factory worker & -0.272 & 0.165 & 2.706 & $0.762(0.551-1.053)$ & 0.100 \\
\hline Medical, scientific, educational staff & -0.474 & 0.205 & 5.347 & $0.623(0.417-0.931)$ & 0.021 \\
\hline Other & -0.076 & 0.160 & 0.227 & $0.927(0.677-1.268)$ & 0.634 \\
\hline \multicolumn{6}{|l|}{ Washing the vulva after sexual intercourse } \\
\hline Yes & & & & 1.000 & \\
\hline No & 0.445 & 0.158 & 7.92 & $1.56(1.144-2.129)$ & 0.005 \\
\hline \multicolumn{6}{|l|}{ Tubal ligation for contraception } \\
\hline No & & & & 1.000 & \\
\hline Yes & 0.295 & 0.126 & 5.529 & $1.343(1.050-1.719)$ & 0.019 \\
\hline \multicolumn{6}{|l|}{ Duration after tubal ligation } \\
\hline$\geq 30$ years & & & & 1.000 & \\
\hline$<30$ years & -0.288 & 0.134 & 4.625 & $0.749(0.576-0.974)$ & 0.032 \\
\hline Average daily dietary folate intake $(\mu \mathrm{g})$ & -0.001 & 0.000 & 8.763 & $0.999(0.998-1)$ & 0.003 \\
\hline
\end{tabular}

OR, odds ratio; CI, confidence interval; CIN, cervical intraepithelial neoplasia.

Table 5. Results of multivariate analysis of the potential risk factors for CIN

\begin{tabular}{|c|c|c|c|c|c|}
\hline Variable & B & S.E. & Wald & OR $(95 \% \mathrm{CI})$ & P value \\
\hline \multicolumn{6}{|l|}{ Age (years) } \\
\hline $56-65$ & -0.407 & & & 1.000 & \\
\hline $46-55$ & -0.487 & 0.160 & 6.475 & $0.666(0.486-0.910)$ & 0.011 \\
\hline $36-45$ & -0.264 & 0.177 & 7.543 & $0.614(0.434-0.870)$ & 0.006 \\
\hline$\leq 35$ & & 0.231 & 1.300 & $0.768(0.488-1.209)$ & 0.254 \\
\hline \multicolumn{6}{|l|}{ Husband's occupation } \\
\hline Farmer & -0.236 & & & 1.000 & \\
\hline Factory worker & -0.475 & 0.172 & 1.895 & $0.789(0.564-1.105)$ & 0.514 \\
\hline Medical, scientific, educational staff & -0.108 & 0.212 & 5.005 & $0.621(0.410-0.943)$ & 0.025 \\
\hline Other & & 0.166 & 0.426 & $0.898(0.648-1.242)$ & 0.169 \\
\hline \multicolumn{6}{|l|}{ Washing the vulva after sexual intercourse } \\
\hline Yes & 0.605 & & & 1.000 & \\
\hline No & -0.001 & 0.166 & 13.349 & $1.831(1.324-2.535)$ & $<0.001$ \\
\hline Average daily dietary folate intake $(\mu \mathrm{g})$ & & 0.000 & 10.012 & $0.999(0.998-0.999)$ & $<0.001$ \\
\hline
\end{tabular}

CIN, cervical intraepithelial neoplasia; OR, odds ratio; $\mathrm{CI}$, confidence interval.

$\mathrm{N}=1036$ including 410 women with CIN lesions and 626 women without CIN lesion. 


\section{Discussion}

This population-based epidemiological study was designed to ensure the uniform training of investigators for data collection through face-to-face interviews and one-to-one questionnaire surveys. Moreover, the closed-ended questions were used for the CIN questionnaire, which could avoid the confounding biases. This current study identified certain potential risk factors that could have contributed to the high incidence of CIN lesions in this geographic area.

\section{Screening for $\mathrm{CIN}$ in the Jiexiu Area}

\section{Cytological testing}

The strength of this study was the large sample size with 20000 subjects. The overall rate of abnormal cytological results was $7.19 \%$, including 6.35\% (1270/20000) among patients with ASC-US, which is within the benchmark data reported by the College of American Pathologists (range, 1.7-9.6\%; median rate, $4.9 \%) .{ }^{18}$ Of a total of $1,270(6.35 \%)$ women with ASC-US Pap test, $21.49 \%(273 / 1270)$ had a diagnosis of CIN1 and $7.05 \%(90 / 1270)$ had a diagnosis of $\mathrm{CIN} 2+$. These values were higher than those reported by López-Alegría $(13 / 446,2.91 \% ; 6.5 \%, 29 / 446) .{ }^{19}$ In addition, AGC was detected in only 2 of $20000(0.01 \%)$ women, which is less than that of the benchmark rate reported by the College of American Pathologists (CAP) (range, $0.0-0.9 \%$; median rate, $0.2 \%) .{ }^{18}$ The very low AGC rate was reported in other studies in China 20, 21, 22 The much low AGC reporting rate compared with CAP benchmarks rate was most likely a reflection of the need for additional medical education on the subject in the cytology laboratories in China.

\section{$\mathrm{CIN}$ and SCC detection}

In the present study, CIN lesion was diagnosed in $2.05 \%$ patients $(95 \% \mathrm{CI}, 1.85-2.25)$; this rate is higher than that in Jingan, Jiangxi Province $(0.46 \%)$ and in Beijing (0.12\%). ${ }^{10} \mathrm{CIN} 1$ was diagnosed in $1.58 \%$ patients $(95 \% \mathrm{CI}, 1.41-1.76)$, which is slightly higher than the rate reported in Jiansu Province, China. ${ }^{23}$ Moreover, CIN2+ ${ }^{+}$was diagnosed in $0.52 \%$ patients (95\% CI, 0.42-0.62) in our study, which is lower than $1.5 \%$ in Xiangyuan County, Shanxi Province, but higher than that in Jingan, Jiangxi Province $(0.36 \%) .{ }^{24}$ The detection rate of SCC was 55/100,000 (95\% CI, 27-99) in the present study, which is notably higher than the cervical cancer rate in China $(9.6 / 100,000)$ reported by the $\mathrm{WHO},{ }^{5}$ and similar to that of SCC $(48 / 100,000)$ in Guangzhou, China, as reported by Zheng in 2015.20 Thus, the high rate of CIN and cervical cancer in Jiexiu area, Shanxi Province, is concerning, and the related risk factors, particularly geographical and population-specific factors, should be further explored in order to develop measures to prevent CIN and cervical cancer.

\section{Risk Factors for CIN in the Jiexiu Area}

\section{Older age and farming as the husband's occupation}

The incidence of CIN lesion was lower in women aged 36-45 years than in those aged 56-65 years, which is different from other studies from the Western countries. The results may be different in the different population. Most reports from the Western countries studied the population with routine cervical cancer screening. However, the most of the women in current study had no previous cervical cancer screening. So the older women had been exposed to the risk factors for cervical cancer for a longer duration. Furthermore, immunity declines gradually after menopause, and the ability to naturally clear HPV infection is lower among women aged $>50$ years. High rates of HPV infection and progression are known to lead to CIN development ${ }^{25}$. Hence, women aged 56-65 years should be the targets of local cervical cancer prevention strategies. Improving the awareness regarding cervical cancer in women in this age group, and the development of cervical cancer screening strategies directly targeting this population may be particularly efficient. Moreover, we observed that women with husbands who were farmers had a higher risk of CIN as compared to women with husbands engaged in occupations associated with higher education levels and in non-manual labor. The CIN risk in women with husbands who worked as medical, scientific, or educational personnel was only $62.1 \%$ of the risk in women with husbands who were farmers. The women with husbands who worked as medical, scientific, or educational staff may have had better health conditions and sexual health awareness as compared to women with husbands who were farmers.

\section{Lack of washing of the vulva after sexual intercourse}

Research in China and worldwide has shown that HR-HPV infection, poor reproductive health, oral contraceptive use, trichomonas vaginitis, and smoking may be risk factors for CIN and cervical cancer. ${ }^{26-28}$ In the present study, we did not identify vaginitis or contraceptive use as risk factors for CIN. However, we observed that the lack of washing of the vulva after sexual intercourse was closely related to the occurrence of CIN. The risk of developing CIN in women who did not wash the vulva after sexual intercourse was 1.831 times greater than the risk in women who did. Since Shanxi Province is an inland area with mountainous areas, drought and water shortage are common. Hence, the bathing conditions 
of the residents tend to be much more limited than those of individuals residing in the southern areas, which have adequate water supply. Some women cleaned the vulva only rarely, and did not clean the vulva after sexual intercourse. Spreading awareness regarding cleaning the vulva after sexual intercourse and promoting sexual health education for women may help prevent precancerous lesions and cervical cancer in this population.

\section{Low dietary folate intake}

In recent years, certain studies have suggested that nutritional factors may play an important role in the CIN development process after HPV infection. ${ }^{29}$ We observed that the dietary staple in the study region was pasta, particularly during winter. During this time, individuals primarily consumed stored foods, such as pasta, cabbage, potatoes, and sauerkraut, and consumed fewer fresh vegetables, animal proteins, and other foods that are rich in folate. Our data indicated that the self-reported amount of dietary folate intake in the CIN group was significantly lower than that of the control group. For every 1- $\mu \mathrm{g}$ increase in the level of average daily dietary folate intake, the rate of CIN was reduced by $0.001 \%$. These results suggest that an increase infolate intake may serve as a protective factor against CIN. Flatley' study showed that a low folate level increased the risk of cervical cancer. ${ }^{30}$ Moreover, Piyathilake recently reported that folate and vitamin B12 may play critical roles in lowering the HPV16 methylation-associated risk of developing higher grades of CIN. ${ }^{31}$ Consistent with these findings, our data indicate that a low dietary folate intake may be a regional risk factor for increased CIN incidence in this area. Therefore, increased dietary folate intake or folic acid supplementation might be used to effectively reduce the incidence and progression of cervical lesions.

In summary, this study provides insight into several specific risk factors associated with increased CIN incidence in the Jiexiu area of Shanxi Province, China. Any future cervical cancer prevention strategies conducted in this area should carefully consider the potential regional risk factors identified in current study. Moreover, future prospective studies will focus on exploring whether supplementation with increased dietary folate intake would decrease the incidence of CIN or cervical cancer in this region.

\section{Acknowledgments}

We gratefully acknowledge the contribution of our coworkers involved in conducting the study and writing this article. We thank all the study participants, as well as the investigators and local healthcare workers from the Jiexiu Family Planning/Women and Children Health Service Center, for their help with the study.

The study was supported by grants from the Special Public Welfare Industry Research of National Health and Family Planning Commission (Beijing, China) (201402010).

\section{Competing Interests}

The authors have declared that no competing interest exists.

\section{References}

1. Torre LA, Bray F, Siegel RL, Ferlay J, Lortet-Tieulent J, Jemal A. Global Cancer Statistics, 2012. Cancer J Clin 2015; 65:87-108.

2. Moy LM, Zhao FH, Li L, et al. Human papillomavirus testing and cervical cytology in primary screening for cervical cancer among women in rural China: comparison of sensitivity, specificity, and frequency of referral. Int J Cancer 2010; 127: 646-656.

3. Zhao FH, Lewkowitz AK, Chen F, et al. Prevalence of human papillomavirus and cervical intraepithelial neoplasia in China: a pooled analysis of 17 population-based studies. Int J Cancer 2012; 131: 2929-2938.

4. TingJ, Kruzikas DT, Smith JS. A global review of age-specific and overall prevalence of cervical lesions. Int J Gynecol Cancer 2010; 20:1244-1249.

5. Bray F, Ren JS, Masuyer E, Ferlay J. Global estimates of cancer prevalence for sites in the adult population in 2008. Int J Cancer 2013; 132: 1133-1145.

6. Di J, Rutherford S, Chu C. Review of the Cervical Cancer Burden and Population-Based Cervical Cancer Screening in China. Asian Pac J Cancer Prev. 2015;16 (17):7401-7.

7. Wang XZ, Liu KR, Yuan FM, Hou JZ, Zhang JJ, Zhang YZ. Analysis of both high incidence of esophageal and cervical cancer in Yangcheng County, Shanxi Province. China Cancer 2011; 4:259-261.

8. Li L, Li LY, Zhu LP, Xun XL, Qi XL. Screening for cervical cancer, breast cancer, and reproductive tract infection in a rural area, Jiangxi Province. China Cancer 2010; 19:797-800.

9. Wang T, Wu MH, Wu YM, Zhang WY. A Population-based Study of Invasive Cervical Cancer Patients in Beijing: 1993-2008. Chin Med J (Engl). 2015; 128(24):3298-304

10. Tao L, Han L, Li X, et al. Prevalence and risk factors for cervical neoplasia: a cervical cancer screening program inBeijing. BMC Public Health 2014; 14:1185.

11. Wang JT, Ma XC, Cheng YY, Ding L, Zhou Q. A case-control study on the association between folate and cervical cancer. Zhonghua Liu Xing Bing XueZaZhi 2006; 27: 424-427.

12. Solomon D, Davey D, Kurman R, et al. The 2001 Bethesda System: terminology for reporting results of cervical cytology. JAMA 2002; 287:2114-2119.

13. Fu GY, Yang HY, Liu ZL, Wang ZY, Jiang P, Wang Z. Study on a multi-test diagnostic kit to diagnose vaginitis. Journal Pathogen Biology 2012; 6:433-435.

14. Nugent R P, Krohn M A, Hillier S L. Reliability of diagnosing bacterial vaginosis is improved by a standardized method of gram stain interpretation. J Clin Microbiol 1991; 29:297-301.

15. Wright TC, Ronnett BM, Kurman RJ. Precancerous lesions of the cervix. In: Kurman RJ, Ellenson LH, Ronnett BM. Blaustein's Pathology of the Female Genital Tract. $6^{\text {th }}$ ed. New York, NY: Springer, 2011: 193-252.

16. Wang J, Zhao W. Application of Simplified Food Frequency Questionnaires on Dietary Assessment. Chinese Journal of Prevention and Control of Chronic Diseases 2000; 8: 29-31.

17. Yang YX, Yang GY, Pan C. China food composition tables 2002. Beijing: Beijing University Medical Press, 2002:79p.

18. Eversole GM, Moriarty AT, Schwartz MR, et al. Practices of participants in the College of American Pathologists Interlaboratory Comparison Program in cervicovaginal cytology, 2006. Arch Pathol Lab Med 2010; 134:331-335.

19. López-Alegría F, Poblete OQ, Lorenzi DS, Oyanedel JC. Clinical management of the first ASC-US report in Chile. Prospective single-cohort study. Sao Paulo Med J 2015; 133:480-487.

20. Zheng B, Austin RM, Liang $X$, et al. Bethesda System reporting rates for conventional Papanicolaou tests and liquid-based cytology in a Large Chinese, College of American Pathologists-verified independent medical laboratory analysis of 1394389 Papanicolaou test reports. Arch Pathol Lab Med 2015; 139:373-377.

21. Tao X, Austin RM, Zhang H, et al. Pap Test Reporting Rates for Conventional Smear and Liquid-Based Cervical Cytology from the Largest Academic Women's Hospital in China: Analysis of 1,248,785 Pap Test Reports. Acta Cytol. 2015;59(6):445-51.

22. Zheng B, Li Z, Liang X, Austin RM, Chen C, Zhao C. Cervical Cytology Reporting Rates from China's Largest College of American Pathologists-Certified Laboratory with a Focus on Squamous Cell Carcinoma Cytology and Its Histopathological Follow-Up Results. Acta Cytol. 2015;59 (5):399-404. 
23. Zhao FH, Zhu FC, Chen W, et al. Baseline prevalence and type distribution of human papillomavirusin healthy Chinese women aged 18-25years enrolled in a clinical trial. Int J Cancer 2014; 135:2604-2611.

24. Ma L, Gu XF, Ma JF, et al. Analysis on the prevalence rate of cervical cancer and screening willingness of rural women from the areas with high prevalence of cervical cancer. Maternal and Child Health Care of China 2012; 27: 5974-5977.

25. Zhang Q, Cao D, Ma Q, Li N, Cui XQ, Yang XF. Natural Outcome of Genital Tract High-risk Human Papillomavirus Infection and Associated Factors among 760 Women. Zhongguo Yi Xue KeXue Yuan Xue Bao 2015; 37:534-540.

26. $\mathrm{Li} \mathrm{CD}, \mathrm{Wu} \mathrm{MH}$, Wang JD, et al. A population-based study on the risks of cervical lesion and human papillomavirus infection among women in Beijing, People's Republic of China. Cancer Epidemiol Biomarkers Prev 2010; 19: 2655-2664.

27. Roura E, Castellsague $\mathrm{X}$, Pawlita M, et al. Smoking as a major risk factor for cervical cancer and pre-cancer: results from the EPIC cohort. Int J Cancer 2014; 135: 453-466.

28. Oh HY, Kim MK, Seo SS, Lee JK. Association of Combined Tobacco Smoking and Oral Contraceptive Use With Cervical Intraepithelial Neoplasia 2 or 3 in Korean Women. J Epidemiol 2016; 26:22-29.

29. Chih HJ, Lee AH, Colville L, Binns CW, Xu D. A review of dietary prevention of human papillomavirus-related infection of the cervix and cervical intraepithelial neoplasia. Nutr Cancer 2013; 65:317-328.

30. Flatley JE, McNeir K, Balasubramani L, Tidy J, Stuart EL, Young TA, Powers HJ. Folate status and aberrant DNA methylation are associated with HPV infection and cervical pathogenesis. Cancer Epidemiol Biomarkers Prev. 2009; 18:2782-2789.

31. Piyathilake CJ, Macaluso M, Chambers MM, et al. Folate and vitamin B12 may play a critical role in lowering the HPV 16 methylation-associated risk of developing higher grades of CIN. Cancer Prev Res. 2014; 7:1128-1137. 